\title{
Enhanced catalytic efficiency and universality of L-amino acid deaminase achieved by a shorter proton transfer distance
}

\author{
Yaoyun $\mathrm{Wu}^{1}$, Sheng Zhang ${ }^{2}$, Wei Song ${ }^{1}$, Jia Liu ${ }^{1}$, Xiulai Chen ${ }^{1}$, Guipeng $\mathrm{Hu}^{1}$, Yiwen \\ Zhou ${ }^{1}$, Liming $\mathrm{Liu}^{3}$, and Jing $\mathrm{Wu}^{1}$ \\ ${ }^{1}$ Jiangnan University \\ ${ }^{2}$ Zhejiang Tianrui Chemical Co., Ltd \\ ${ }^{3}$ Jiangnan Univ.
}

May 31, 2021

\begin{abstract}
L-amino acid deaminase (LAAD, EC 1.4.3.2) catalyzes the deamination of $\alpha$-amino acids. At present, sustainable enzymatic $\alpha$-keto acids synthesis remains limited by the low catalytic efficiency of wild-type LAADs. In this study, catalytic mechanism was elucidated, and catalytic distance D1 between the substrate $\alpha \mathrm{C}-\mathrm{H}$ and the cofactor FAD N(5) was identified as the key factor limiting efficiency of Proteus mirabilis PmiLAAD. Shortening the distance via protein engineering improved catalytic efficiency toward six selected amino acids. The two variants with the best catalytic properties were W1, which exhibited a preference for short-chain aliphatic amino acids and charged amino acids, and W2, which showed a preference for large aromatic amino acids and sulfur-containing amino acids. The mutated residues in the two variants altered the binding pose of the substrate, $\alpha$-hydrogen was improved to be more perpendicular against the plain of the isoalloxazine ring causing the angle between the substrates' $\alpha \mathrm{C}-\mathrm{H}$, FAD N(5), and FAD N(10) to approach $90^{\circ}$, and thus shortened the distance. Finally, W1 and W2 were cascade in one Escherichia coli cell to obtain strain S3, which exhibited conversion $>90 \%$ and yield $>100 \mathrm{~g} / \mathrm{L}$ toward all selected substrates. These results provide the basis for improving industrial production of $\alpha$-keto acids via microbial deamination of $\alpha$-amino acids.
\end{abstract}

Enhanced catalytic efficiency and universality of L-amino acid deaminase achieved by a shorter proton transfer distance

Yaoyun $\mathrm{Wu}^{1,2}$, Sheng Zhang ${ }^{3}$, Wei Song ${ }^{1,2}$, Jia $\mathrm{Liu}^{2}$, Xiulai $\mathrm{Chen}^{2}$, Guipeng $\mathrm{Hu}^{1,2}$, Yiwen Zhou ${ }^{1}$, Liming $\mathrm{Liu}^{2}$, Jing $\mathrm{Wu}^{1 *}$

${ }^{1}$ School of Pharmaceutical Science, Jiangnan University, Wuxi 214122, China

${ }^{2}$ State Key Laboratory of Food Science and Technology, Jiangnan University, Wuxi 214122, China

${ }^{3}$ Tianrui Chemical Co., Ltd Department of Chemistry Quzhou 324400, China

*Corresponding author.

Mailing address: School of Pharmaceutical Science, Jiangnan University, 1800 Lihu Road, Wuxi 214122, China

Fax/Tel.: +86-510-85915657

E-mail: wujing@jiangnan.edu.cn

\section{ABSTRACT}


L-amino acid deaminase (LAAD, EC 1.4.3.2) catalyzes the deamination of $\alpha$-amino acids. At present, sustainable enzymatic $\alpha$-keto acids synthesis remains limited by the low catalytic efficiency of wild-type LAADs. In this study, catalytic mechanism was elucidated, and catalytic distance D1 between the substrate $\alpha \mathrm{C}-\mathrm{H}$ and the cofactor FAD N(5) was identified as the key factor limiting efficiency of Proteus mirabilisPmi LAAD. Shortening the distance via protein engineering improved catalytic efficiency toward six selected amino acids. The two variants with the best catalytic properties were W1, which exhibited a preference for short-chain aliphatic amino acids and charged amino acids, and W2, which showed a preference for large aromatic amino acids and sulfur-containing amino acids. The mutated residues in the two variants altered the binding pose of the substrate, $\alpha$-hydrogen was improved to be more perpendicular against the plain of the isoalloxazine ring causing the angle between the substrates' $\alpha \mathrm{C}-\mathrm{H}, \mathrm{FAD} \mathrm{N}(5)$, and FAD N(10) to approach $90^{\circ}$, and thus shortened the distance. Finally, W1 and W2 were cascade in one Escherichia coli cell to obtain strain S3, which exhibited conversion $>90 \%$ and yield $>100 \mathrm{~g} / \mathrm{L}$ toward all selected substrates. These results provide the basis for improving industrial production of $\alpha$-keto acids via microbial deamination of $\alpha$-amino acids.

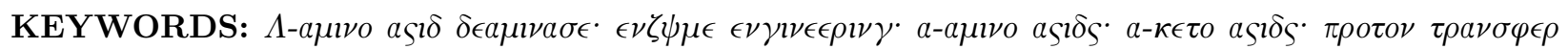
$\delta i \sigma \tau a \nu_{S} \epsilon$ INTRODUCTION

$\alpha$-Keto acids contain both a carboxyl and a keto bifunctional group(Cooper et al. 1983; .Li et al. 2019). They are widely used in pharmaceutical(Wang et al. 2019), feed(Manjarinet al. 2020), food(Song et al. 2016), and chemical(Cooperet al. 1983; Ogo et al. 2004; Xie et al. 2019) synthesis. For instance, $\alpha$-keto acid tablets can be used as therapeutic agents against nephropathy (e.g., uremia and nitrogen accumulation disorders)(Wang et al. 2019), as feed additive (e.g., $\alpha$-ketoisocaproic or $\alpha$-ketoisovaleric acid) to stimulate the growth of animal muscle(Manjarin et al. 2020), as precursor in indole-3-acetic acid synthesis(Song et al. 2016), and for the production of chemicals (e.g., heterocyclic compounds(Ogo et al.2004), n-butanol(Ayodele et al. 2020), and D-amino acids(Liuet al. 2020)). Consequently, chemical(Cooper et al. 1983; Jin et al. 2021; Zhao et al. 2020), fermentative(Vogtet al. 2015), and biocatalytic methods(Jambunathan et al.2014; Liu et al. 2013; Pei et al. 2020) have been developed to ensure a sufficient supply of $\alpha$-keto acids to meet market demand. Biocatalytic methods have attracted particular attention owing to their short reaction period, high conversion rates, and environmental-friendly processes.

Currently, enzymatic synthesis of $\alpha$-keto acids relies on the use of amino acid transaminase (AAT, EC 2.1.1.X)(Taylor et al. 1998; Wuet al. 2018), L-amino acid dehydrogenase (LAADH, EC 1.4.1.5)(Jambunathan et al. 2014; Wakamatsu et al. 2017), amino acid oxidase (DAAO, EC 1.1.3.3; LAAO, EC 1.4.3.3)(Asano et al. 2019; Hossain et al. 2014a; Mattevi et al. 1996; Nakano et al. 2019), and Lamino acid deaminase (LAAD, EC 1.4.3.2)(Ju et al. 2017; Ju et al. 2016; Liu et al.2013; Molla et al. 2017; Motta et al. 2016; Pei et al. 2020; Song et al. 2016; Wang et al. 2019; Wu et al. 2020) (Schemes 1 and 2). However, all of these enzymes suffer from considerable limitations: AAT and LAADH present low conversion rates due to simultaneous reversible reactions; AAT-catalyzed transamination requires additional amino acceptors (e.g., $\alpha$-ketoglutaric acid)(Tayloret al. 1998); LAADH requires NAD, a costly compound, as a cofactor(Wakamatsu et al. 2017), as well as complex separation and purification of the products; and LAAO-catalyzed deamination releases toxic byproducts (e.g., $\mathrm{H}_{2} \mathrm{O}_{2}$ ) and the difficulties in its recombinant production(Hossain et al.2014a); DAAT and DAAO can also produce keto acids, but the substrates involved are expensive unnatural amino acids. Therefore, these enzymes are not suitable for the industrial production of $\alpha$-keto acids. In contrast, LAAD-catalyzed deamination reactions do not require any additional cofactors or amino receptors, and do not release toxic by-products. The crystal structure (Pma LAAD (Motta et al.2016) and $P v$ LAAD (Ju et al. 2016)) first reported laid the foundation for later mechanistic elucidation and protein engineering. It was found that the structure of LAAD was composed of three parts: substrate binding domain (SBD), FAD binding domain gate (FBD) and insertion module(Molla et al. 2017). On this basis, various LAAD variants have been engineered. Li et al. developed a one-step process for KIV production via expressing P. myxofaciens LAAD in E. coli BL21(DE3) and enhanced the yield of KIV (8.2 g/L) by site-directed saturation mutagenesis of LAAD to obtain mutants F318T and N100H (Liet al. 2017). Mutant K104R was screened from $P$. vulgarisLAAD mutant libraries constructed by epPCR for a 1.3-fold increase 
in KMTB enzymatic activity, but the yield of KMTB was only $63.6 \mathrm{~g} / \mathrm{L}$ (Hossainet al. 2014b). The Proteus mirabilis Pmi $\mathrm{LAAD}^{\mathrm{T} 436 / \mathrm{W} 438 \mathrm{~A}}$ variant presents an enlarged entrance $(1.71 \AA)$ to the access tunnel, which increases its catalytic efficiency toward L-Ile to $98.9 \mathrm{~g} / \mathrm{L}$ (99.7\% conversion), but this variant only works well for L-Ile(Yuan et al. 2019). ThePmi LAAD ${ }^{\mathrm{F} 93 \mathrm{~S} / \mathrm{P} 186 \mathrm{~A} / \mathrm{M} 394 \mathrm{~V} / \mathrm{F} 184 \mathrm{~S}}$ variant achieved a 6.6 -fold higher specific activity toward L-Phe compared to the wild-type, but substrate loading was limited to $12 \mathrm{mM}(2 \mathrm{~g} / \mathrm{L})($ Wuet al. 2020). As can be seen, there is room for further improving LAAD catalytic efficiency and in particular, for gearing it toward more than one substrate. Overall, protein engineering should focus on developing more efficient and general-purpose LAAD variants.

In the present study, an efficient and universal whole-cell catalyst for $\alpha$-keto acids production, with excellent catalytic efficiency and wide substrate scope, was constructed through rational protein engineering and a multi-enzyme cascade strategy. First, the catalytic mechanism of LAAD was analyzed and the key factors affecting catalytic efficiency were identified. Then, a protein engineering method, coupled with screening, allowed for the selection of two variants with high catalytic efficiency toward different amino acids. Finally, a one-pot two-enzyme cascade strategy was applied by co-expressing the two variant LAADs inEscherichia coli .

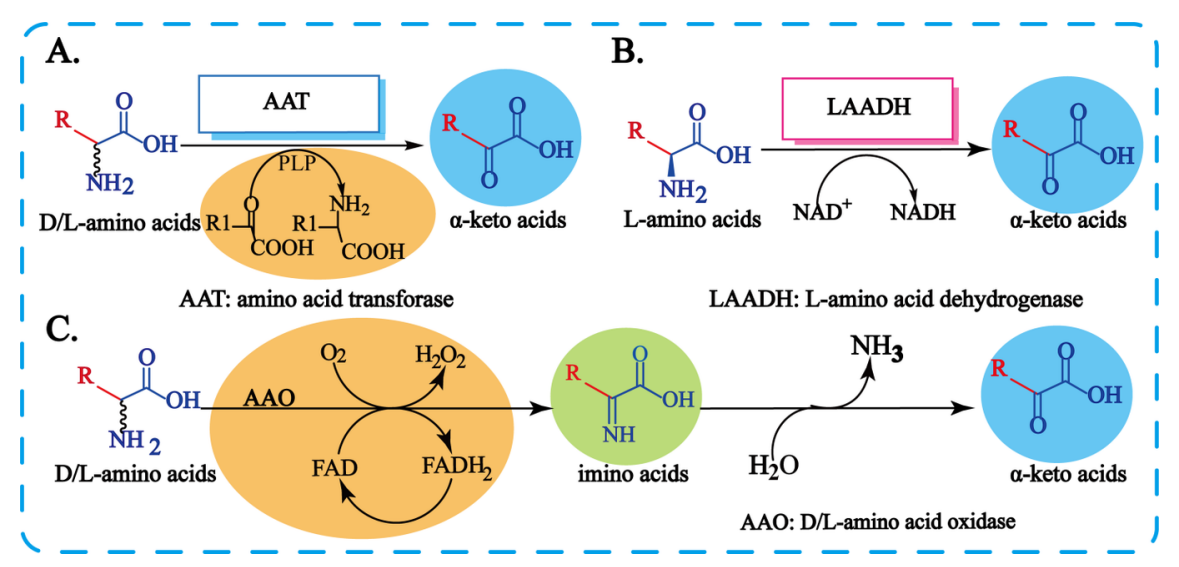

Scheme 1. Deamination reactions of Amino acid transaminase (AAT), L-amino acid dehydrogenase (LAADH), and L-amino acid oxidase (AAO). 


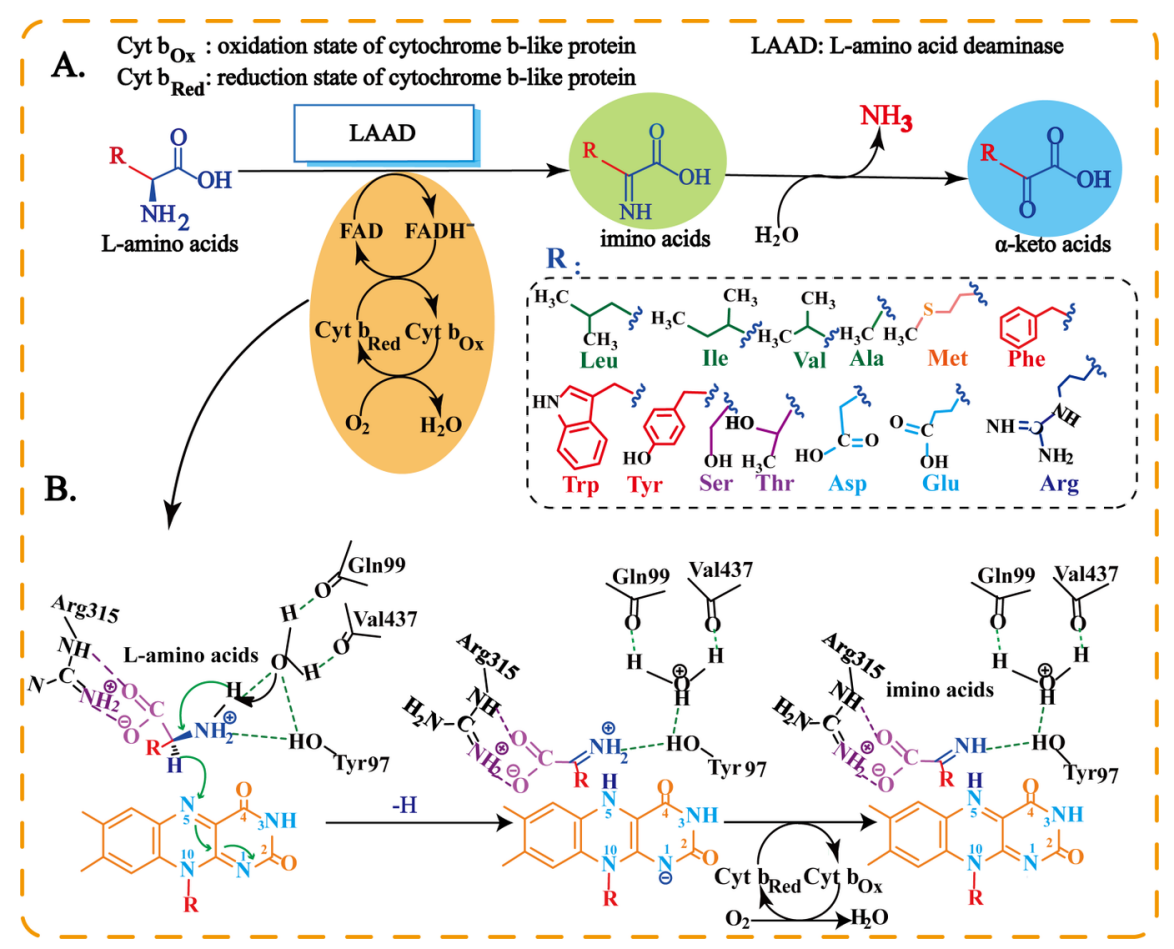

Scheme 2. Deamination reactions of L-amino acid deaminase (LAAD). (A) LAAD overall catalytic pathway diagram and the structural diagram of $\mathrm{R}$ substituents. (B) LAAD process diagram catalyzing substrate proton transfer.

\section{RESULTS}

\section{Substrate scope of PmiLAAD}

The catalytic efficiency of Pmi LAAD toward 13 L-amino acids was investigated and the results are illustrated in Supplementary Table 1. Efficiency was dictated by the amino acids' R groups: the highest catalytic activity was observed in long-chain aliphatic amino acids, such as L-Leu (100\%) and L-Ile (96.9\%), followed by the sulfur-containing L-Met (73.5\%), short-chain aliphatic L-Val (66.3\%), aromatic L-Phe (54.1\%), positively charged L-Arg (28.2\%), and negatively charged L-Glu (8.6\%). Hardly no catalytic activity was detected toward amino acids containing a hydroxyl group, such as L-Ser and L-Thr. Six amino acids with diverse R groups (L-Leu, L-Met, L-Val, L-Phe, L-Arg, and L-Glu) were selected for further experiments. When 100 $\mathrm{g} / \mathrm{L}$ of L-Leu, L-Met, L-Val, L-Phe, L-Arg, and L-Glu were transformed by $10 \mathrm{~g} / \mathrm{L}$ of whole-cell catalyst (Pmi LAAD), the titer of the corresponding $\alpha$-keto acids was 98.2, 72.1, 65.3, 53.2, 27.6, and $8.4 \mathrm{~g} / \mathrm{L}$, respectively (Figure 1B). These values indicated that there was space for improving Pmi LAAD catalytic efficiency through protein engineering.

\section{The catalytic mechanism of PmiLAAD}

To effectively guide the protein engineering of Pmi LAAD, we relied on the hydrogen transfer mechanism of flavin-dependent enzymes(Mattevi et al. 1996; Molla et al. 2017; Mottaet al. 2016; Sandoval et al. 2021; Umhau et al.2000). According to this hypothetical mechanism, Pmi LAAD first transfers an hydride ion (one proton and 2 electrons) of the substrate, from the $\alpha$-carbon $(\alpha \mathrm{C}-\mathrm{H})$ to the cofactor FAD. As shown in Scheme $2 \mathrm{~B}$, the $\alpha \mathrm{C}-\mathrm{H}$ is transferred to the FAD isoalloxazine ring $\mathrm{N}(5)$, thus forming the anionic form of reduced FAD $\left(\mathrm{FADH}^{-}\right)$; while the $\mathrm{NH}_{3}{ }^{+}-\mathrm{H}$ is accepted by the active site water molecule. The transfer of the hydride is accompanied by transformation of the L-amino acids to an imino acids. Next, the H-atoms are transferred from $\mathrm{FADH}^{-}$to an $\mathrm{O}$-atom through cytochrome b-like proteins, thereby regenerating $\mathrm{FAD}$ and 
releasing $\mathrm{H}_{2} \mathrm{O}$. In the meantime, the imino acids undergoes spontaneous hydrolysis to $\alpha$-keto acids in aqueous solution and ammonia was released (Scheme 2A). Given that $\alpha \mathrm{C}-\mathrm{H}$ of the substrate participate in $\mathrm{H}$-atom transfer from $\alpha \mathrm{C}-\mathrm{H}$ to FAD N(5), we speculated that the distance between $\alpha \mathrm{C}-\mathrm{H}$ and FAD N(5)(Molla et al. 2017; Williamset al. 2000)(called catalytic distance D1) might be the key factor affecting Pmi LAAD catalytic efficiency.

To determine how D1 affected Pmi LAAD catalytic efficiency, a homology model of Pmi LAAD was built using Proteus myxofaciens LAAD (Pma LAAD) as template (PDB ID: 5fjn)(Mottaet al. 2016) (Figure 2). The model was docked with the six selected amino acid substrates and then subjected to kinetic simulations to evaluate the various binding conformations. Accordingly, D1 of Pmi LAAD was estimated as: D1 Leu $(2.4 \AA)$ $<\mathrm{D} 1_{\text {Met }}(2.8 \AA)<\mathrm{D} 1_{\text {Val }}(2.9 \AA)<\mathrm{D} 1_{\text {Phe }}(3.0 \AA)<\mathrm{D} 1_{\text {Arg }}(3.3 \AA)<\mathrm{D} 1_{\text {Glu }}(3.8 \AA)$. (The detailed process of homology modeling and molecular docking and the method used to measure D1 can be found in Supporting Information.) The relative enzymatic activity of Pmi LAAD decreased with increasing D1: L-Leu (100\%) $>$ L-Met $(73.5 \%)>$ L-Val $(66.3 \%)>$ L-Phe $(54.1 \%)>$ L-Arg $(28.2 \%)>$ L-Glu (8.6\%) (Figure 1C). The negative correlation between catalytic efficiency and D1 could be explained by a short D1 facilitating proton transfer and consequently increasing catalytic efficiency. Therefore, shortening D1 by protein engineering could potentially improve Pmi LAAD catalyticy efficiency.

A.

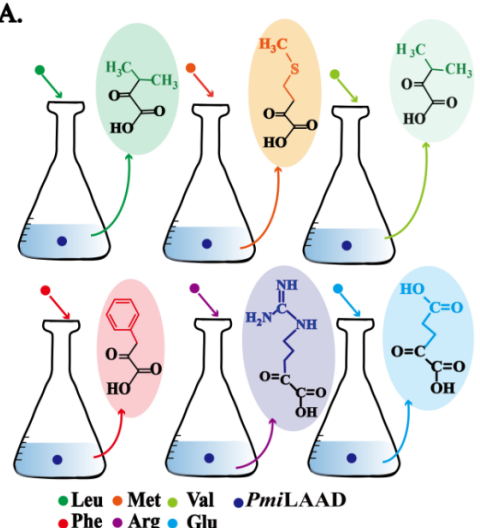

C.

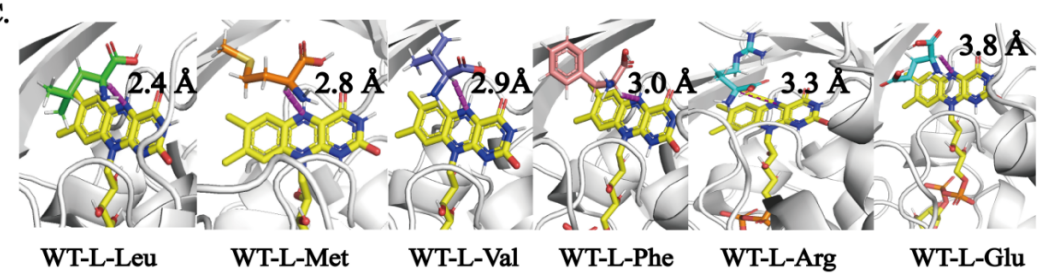

B.

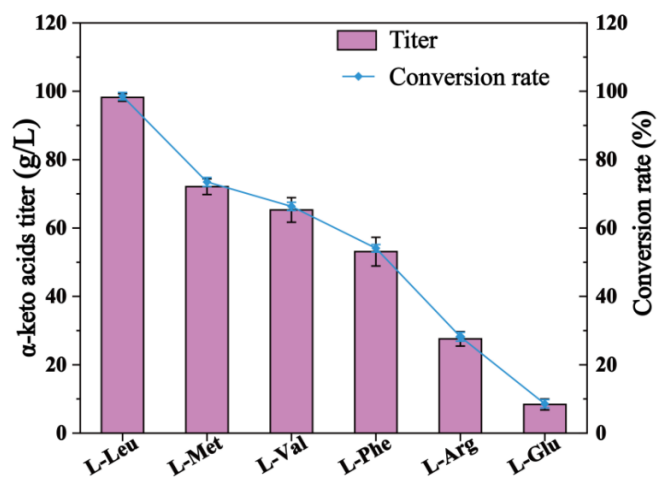

$3 \AA$

WT-L-Leu

WT-L-Me

WT-L-Val WT-L-Phe WT-L-Arg

$$
\text { . }
$$

Figure 1. Representative amino acids conversion of wild-type strains and D1 analysis of representative substrates in relation to Pmi LAAD ${ }^{\mathrm{WT}}$ model. 


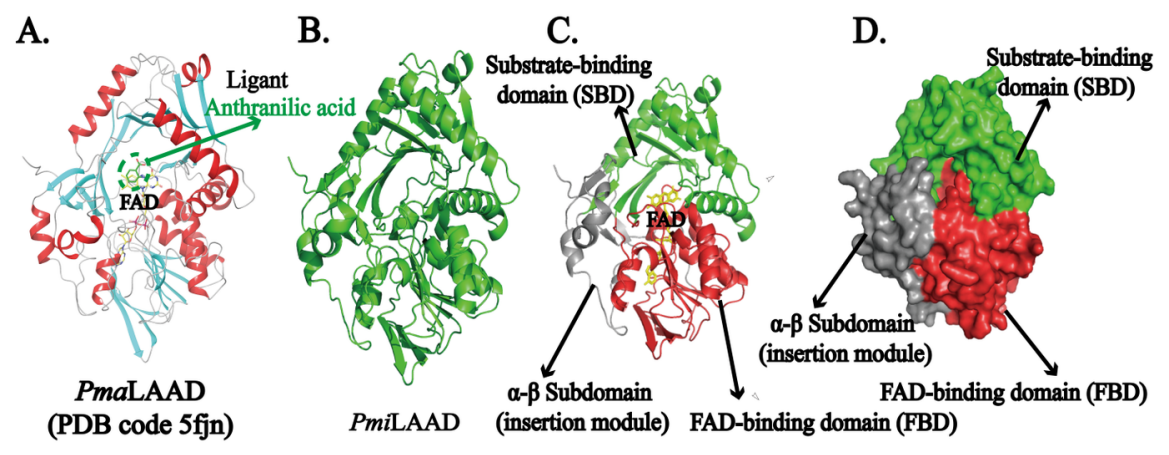

Figure 2. Structure diagram of Pmi LAAD. (A, B) The homology model of Pmi LAAD built based on the crystal structure of LAAD from Proteus myxofaciens (PDB ID: 5fjn); (C) Docking the cofactor FAD into this model, The green, red, and gray cartoon structures represent the substrate binding domain, cofactor binding domain, and insertion module, respectively; (D) The surface mode of the domain distribution map of Pmi LAAD. The yellow (green) stick model represents the cofactor FAD and Ligant (Anthranilic acid, inPma LAAD).

\section{Protein engineering to increase the catalytic efficiency of $P m i$ LAAD}

The substrate and FAD binding domains were considered to be the key factors affecting D1. Nine potential amino acid residues in these two regions (S98, D149, K150, P203, V276, L278, S279, H295, and H301) were selected and subjected to alanine scanning (Supplementary Figure 1). S98A and D149A emerged as the ones causing the greatest increase in relative activity toward Val, Arg, and Glu: 13.6\%, 22.3\%, and $34.2 \%$ (S98A), and 10.1\%, 14.3\%, and 30.0\% (D149A), respectively (Supplementary Figure 2A). Saturation of mutagenesis yielded the single variants S98A and D149Y, whose relative activity toward Val, Arg, and Glu had increased by $23.2 \%, 22.6 \%$, and $21.4 \%$, respectively (Supplementary Table 2). Then, these two variants were combined to obtain Pmi LAAD ${ }^{\mathrm{S} 98 \mathrm{~A} / \mathrm{D} 149 \mathrm{Y}}$ (W1) (Supplementary Figure 3), which significantly improved the catalytic efficiency toward short-chain aliphatic amino acids (L-Val, 85.8\%) or charged amino acids (L-Arg, 65.1\%; L-Glu, 51.7\%), but not toward large-volume aromatic L-Phe or sulfur-containing LMet (Table 1). Docking simulation results revealed a stronger hydrophobic interaction and van der Waals forces between the substrate channel and the side chain of L-Phe or L-Met, which likely affected D1. Therefore, ten dynamic unstable amino acid residues, i.e., the residues whose RMSFs values fluctuates greatly (T105, I109, F110, D144, E145, K146, F317, E340, S412, and E417) near the substrate channel were selected through conformational kinetics (Supplementary Figure 4) and subjected to alanine scanning. T105A, D144A, E145A, E340A, S412A, and E417A emerged as the ones increasing the relative activity toward Phe and Met by [?]10\% (Supplementary Figure 2B). Finally, the six variants were combined to generate Pmi LAAD $^{\mathrm{T} 105 \mathrm{~A} / \mathrm{D} 144 \mathrm{~A} / \mathrm{E} 145 \mathrm{~A} / \mathrm{E} 340 \mathrm{~A} / \mathrm{S} 412 \mathrm{~A} / \mathrm{E} 417 \mathrm{~A}}$ (W2) (Supplementary Figure 5), which exhibited higher catalytic efficiency toward bulkier substrates, including L-Phe (165.9\%), L-Trp (151.5\%), L-Tyr (155.8\%), and L-Met (123.9\%), compared to Pmi LAAD (Table 1).

Table 1. Comparison table of transformation of strain S3 with WT, variants W1 and W2.

\begin{tabular}{lllllllll}
\hline $\begin{array}{l}\text { Strains } \\
\text { Substrate }\end{array}$ & WT & WT & W1 & W1 & W2 & W2 & S3 & S3 \\
& (g/L) & C. $(\%)$ & $\begin{array}{l}\text { Yield } \\
(\mathrm{g} / \mathrm{L})\end{array}$ & C. $(\%)$ & $\begin{array}{l}\text { Yield } \\
(\mathrm{g} / \mathrm{L})\end{array}$ & $\begin{array}{l}\text { C. }(\%) \\
\text { Yield }\end{array}$ & $\begin{array}{l}\text { C. }(\%) \\
(\mathrm{g} / \mathrm{L})\end{array}$ \\
L-Leu & $98.2 \pm 1.3$ & $98.5 \pm 1.4$ & $99.5 \pm 1.2$ & $99.8 \pm 1.1$ & $99.5 \pm 1.3$ & $99.7 \pm 1.4$ & $99.7 \pm 1.2$ & $99.8 \pm 1$. \\
L-Ile & $95.6 \pm 1.0$ & $97.6 \pm 1.6$ & $97.9 \pm 1.4$ & $98.8 \pm 1.3$ & $97.6 \pm 1.1$ & $99.6 \pm 1.2$ & $99.4 \pm 1.4$ & $99.5 \pm 1$. \\
L-Met & $72.1 \pm 1.6$ & $73.5 \pm 1.7$ & $78.1 \pm 1.1$ & $79.7 \pm 1.2$ & $89.4 \pm 1.5$ & $91.2 \pm 1.8$ & $92.1 \pm 1.7$ & $93.9 \pm 1$. \\
& & & & & & $(123.9 \%)$ & & \\
L-Val & $65.3 \pm 1.4$ & $66.3 \pm 1.8$ & $84.1 \pm 1.2$ & $85.8 \pm 1.5$ & $67.9 \pm 1.3$ & $69.3 \pm 1.4$ & $90.7 \pm 1.6$ & $92.6 \pm 1$.
\end{tabular}




\begin{tabular}{lllllllll} 
L-Ala & $56.62 \pm 1.2$ & $59.6 \pm 1.6$ & $74.4 \pm 1.3$ & $75.9 \pm 1.4$ & $57.6 \pm 1.2$ & $58.8 \pm 1.7$ & $78.7 \pm 1.4$ & $80.3 \pm 1$ \\
L-Phe & $53.2 \pm 1.3$ & $54.1 \pm 1.4$ & $53.9 \pm 1.2$ & $55.2 \pm 1.6$ & $88.1 \pm 1.8$ & $89.9 \pm 1.6$ & $88.9 \pm 1.5$ & $90.7 \pm 1$ \\
& & & & & & $(165.9 \%)$ & & \\
L-Trp & $36.9 \pm 1.5$ & $38.9 \pm 1.7$ & $39.1 \pm 1.2$ & $39.9 \pm 1.4$ & $57.7 \pm 1.5$ & $58.8 \pm 1.2$ & $60.7 \pm 1.4$ & $61.9 \pm 1$ \\
& & & & & & $(151.5 \%)$ & & \\
L-Tyr & $29.5 \pm 1.2$ & $30.1 \pm 1.3$ & $30.5 \pm 1.6$ & $31.1 \pm 1.7$ & $46.2 \pm 1.4$ & $46.9 \pm 1.3$ & $50.5 \pm 1.6$ & $51.6 \pm 1$ \\
& & & & & & $(155.8 \%)$ & & \\
L-Arg & $27.6 \pm 1.5$ & $28.2 \pm 1.7$ & $65.1 \pm 1.4$ & $66.4 \pm 1.8$ & $30.1 \pm 1.6$ & $30.7 \pm 1.2$ & $88.6 \pm 1.5$ & $90.4 \pm 1$ \\
L-Asp & $15.8 \pm 1.4$ & $16.2 \pm 1.3$ & $63.8 \pm 1.6$ & $65.1 \pm 1.7$ & $16.2 \pm 1.5$ & $16.8 \pm 1.7$ & $78.2 \pm 1.4$ & $79.5 \pm 1$ \\
L-Glu & $8.4 \pm 1.8$ & $8.6 \pm 1.6$ & $50.7 \pm 1.5$ & $51.7 \pm 1.3$ & $10.4 \pm 1.6$ & $10.6 \pm 1.9$ & $87.4 \pm 1.5$ & $90.1 \pm 1$ \\
L-Ser & n.d. & n.d. & $2.7 \pm 1.3$ & $2.8 \pm 0.6$ & $0.9 \pm 0.3$ & $1.0 \pm 0.3$ & $5.8 \pm 1.1$ & $5.9 \pm 0.9$ \\
L-Thr & $0.4 \pm 0.2$ & $0.4 \pm 0.1$ & $2.6 \pm 1.2$ & $2.7 \pm 0.7$ & $0.7 \pm 0.2$ & $0.8 \pm 0.1$ & $5.5 \pm 0.8$ & $5.6 \pm 1.0$ \\
\hline
\end{tabular}

Note: Reactions were performed in triplicate with resting cells of WT, W1, W2, or S3 (10 g/L wet cells) and 13 L-amino acids (100 g/L; Leu, L-Ile, L-Met, L-Val, L-Ala, L-Phe, L-Trp, L-Tyr, L-Arg, L-Asp, L-Glu, L-Ser or L-Thr) in $20 \mathrm{~mL}$ Tris- $\mathrm{HCl}$ buffer $(20 \mathrm{mM}, \mathrm{pH} 8.0)$ at $200 \mathrm{rpm}$ and $25{ }^{\circ} \mathrm{C}$ for $24 \mathrm{~h}$. The abbreviation C. stands for conversion. The percentages in parentheses are the fold change in conversion rate of the variant compared to the parentalPmi LAAD, and only the data that are significantly elevated are indicated.

\section{Evaluation of W1 and W2 performance and analysis of the enhancement mechanism}

The catalytic properties of variant W1 toward different amino acids were evaluated. As illustrated in Supplementary Table 4, W1 significantly increased catalytic efficiency toward L-Val, L-Arg, and L-Glu; while only slightly improving catalysis of the other substrates. D1 toward Val decreased the most (24.1\%; from 2.9 to $2.4 \AA$ ), resulting in $k_{c a t}, K_{m}, k_{\text {cat }} / K_{m}$, and specific activity increasing by $71.4 \%, 31.2 \%, 29.3 \%$, and $86.1 \%$, respectively. Shortening of D1 toward Arg (9.1\%) and Glu (5.3\%) led to an increase in $k_{\text {cat }}(53.8 \%$ and $52.5 \%), K_{m}(28.5 \%$ and $33.4 \%), k_{c a t} / K_{m}(25.1 \%$ and $23.2 \%)$, and specific activity $(130.3 \%$ and $584.8 \%)$.

To identify the mechanisms underlying improved catalytic efficiency, the interaction force, substrate binding pose, and angle were compared. When S98 and D149 (hydrophobicity, -0.8 and -3.5) were mutated to A98 and Y149 (hydrophobicity, +1.8 and -1.3), the pocket hydrophobicity was increased. This change altered the hydrophobic interactions between S98 and substrate- $\mathrm{COOH}_{\text {, }}$ and between Y149-OHand substrate-NH2 (Figure 3 ), $\alpha$-hydrogen was improved to be more perpendicular against the plain of the isoalloxazine ring causing the angle $\vartheta$ between the substrates' $\alpha \mathrm{C}-\mathrm{H}, \mathrm{FAD} \mathrm{N}(5)$, and FAD N(10) to approach $90^{\circ}$. The shortest D1 occurs when the substrate's $\alpha \mathrm{C}-\mathrm{H}$ is perpendicular to the FAD isoalloxazine ring. Interestingly, we found that $\vartheta$ of $\mathrm{W} 1_{\text {Val }}$ decreased from $131.2^{\circ}$ to $91.2^{\circ}$, W1 $\mathrm{Arg}$ from $142.2^{\circ}$ to $93.4^{\circ}$, and $\mathrm{W} 1_{\mathrm{Glu}}$ from $148.2^{\circ}$ to $98.2^{\circ}$, which resulted in $\mathrm{D} 1_{\mathrm{Val}}, \mathrm{D} 1_{\mathrm{Arg}}$, and $\mathrm{D} 1_{\mathrm{Glu}}$ to be reduced by $0.5,0.3$, and $0.2 \AA$, respectively (Figure 4). The shorter D1 in W1 led to an increase in catalytic efficiency toward L-Val, L-Arg, and L-Glu. Notably, $\vartheta$ and D1 remained almost unchanged with respect to L-Leu, L-Phe, and L-Met, possibly explaining why the catalytic efficiency of W1 toward these amino acids had not improved. 
A.
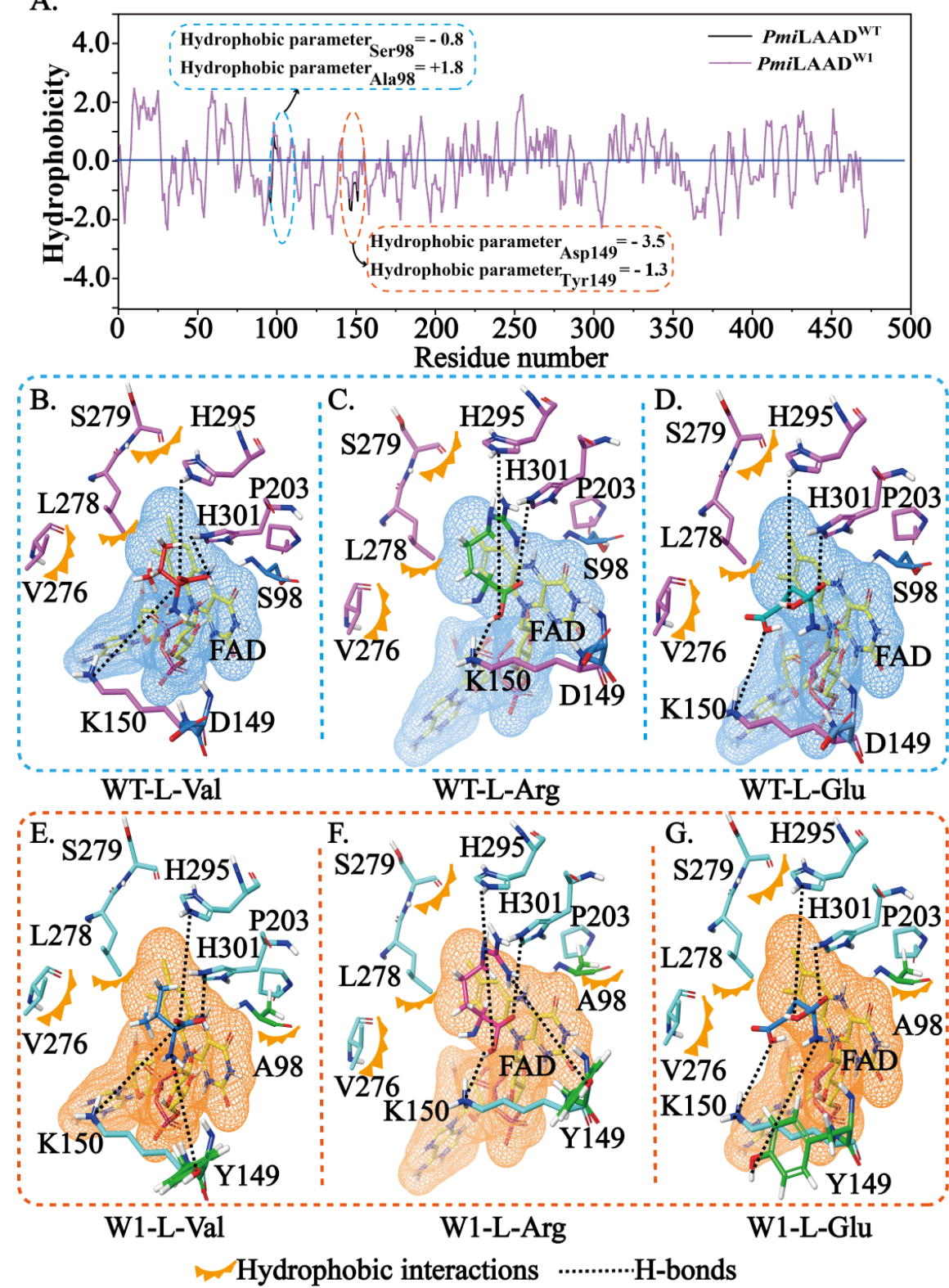

Figure 3. Hydrophobicity and interaction analysis of $P m i$ LAAD ${ }^{\mathrm{WT}}$ and variant W1. (A) Comparison of hydrophobicity of corresponding amino acid residues between Pmi LAAD ${ }^{\mathrm{WT}}$ and variant W1. The black lines represent the Pmi LAAD ${ }^{\mathrm{WT}}$, and the purple lines represent the variant W1. (B-D) Interaction analysis of substrates (L-Val, L-Arg and L-Glu) with Pmi LAAD ${ }^{\mathrm{WT}}$. (E-G) Interaction analysis of substrates (L-Val, L-Arg and L-Glu) with W1. 
A.

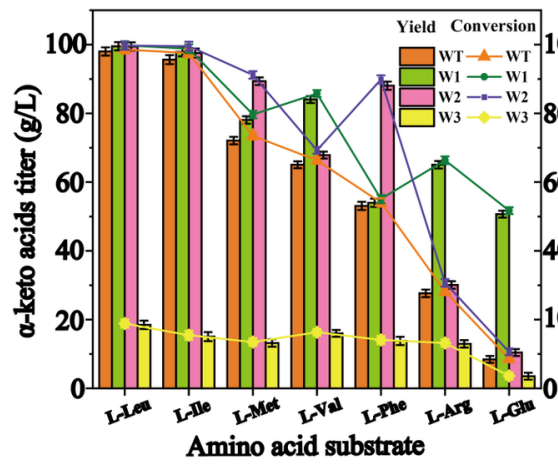

D.

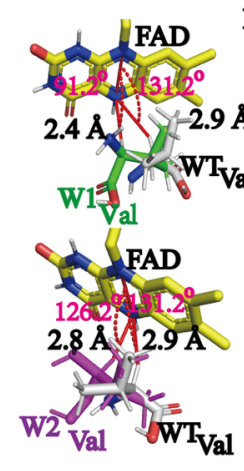

E.

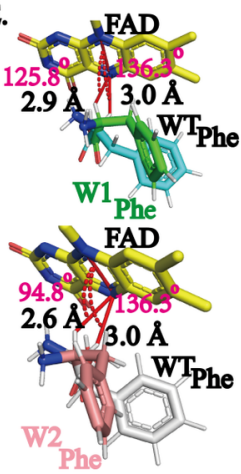

B.

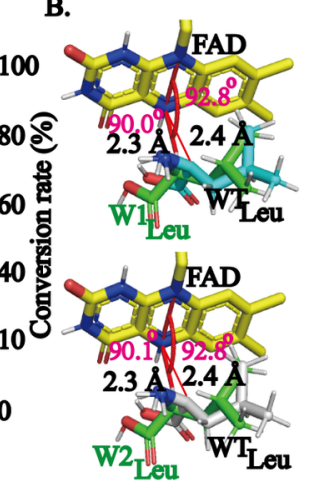

F.

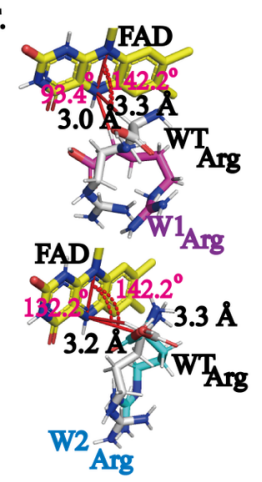

C.

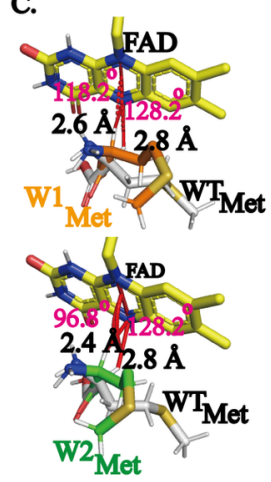

G.

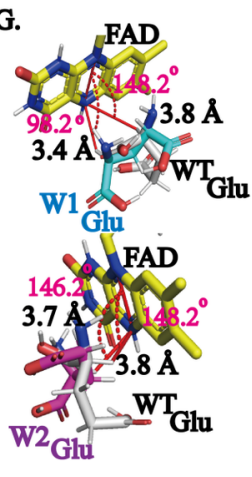

Figure 4. Representative amino acids transformations of variants W1-W3 and binding pose analysis in the docking model.(A) Representative amino acids transformation results of variants W1-W3. (B-G) Posture analysis of variant $\mathrm{W} 1$ or $\mathrm{W} 2$ after docking with representative substrates (L-Leu, L-Met, l-val, L-Phe, L-Arg or L-Glu).

We also evaluated the properties of variant W2 for different amino acids. As shown in Supplementary Table 3 , variant W2 significantly increased catalytic efficiency toward L-Phe and L-Met, but not toward the other substrates. D1 1 Phe decreased the most (15.4\%; from 3.0 to $2.6 \AA$ ), which increased its $k_{\text {cat }}, K_{m}, k_{\text {cat }} / K_{m}$, and specific activity toward L-Phe by $107.1 \%, 23.5 \%, 64.7 \%$, and $65.5 \%$, respectively. D1 $1_{\mathrm{Met}}$ decreased by $14.7 \%$ (from 2.8 to $2.4 \AA$ ), which increased $k_{\text {cat }}, K_{m}, k_{\text {cat }} / K_{m}$, and specific activity by $57.7 \%, 26.8 \%, 23.8 \%$, and $38.2 \%$, respectively.

The mechanisms underlying improved catalytic efficiency of variant W2 were analyzed. Compared to Pmi LAAD, molecular dynamic simulations revealed an increase in root-mean-square fluctuations (RMSFs) for six residues (T105, D144, E145, E340, S412, and E417) around the substrate channel (Figure 5). The increased conformational dynamic of these mutated residues resulted in greater structural flexibility of the channel(W. Song et al. 2020; Yang et al. 2017), which changed the orientation of the substrate side-chain (phenyl or methylthio group) (Figure $4 \mathrm{C}$ and $4 \mathrm{E}$ ). As a consequence, the binding posture of L-Phe and L-Met was significantly altered, decreasing $\vartheta$ of $\mathrm{W} 2_{\mathrm{Phe}}$ and $\mathrm{W} 2_{\mathrm{Met}}$ from $136.3^{\circ}$ to $94.8^{\circ}$ and from $128.2^{\circ}$ to $96.8^{\circ}$, respectively. The reduced $\vartheta$ shortened $\mathrm{D} 1_{\mathrm{Phe}}$ and $\mathrm{D} 1_{\mathrm{Met}}$ by $0.4 \AA$, resulting in higher catalytic efficiency toward L-Phe and L-Met. Meanwhile, $\vartheta$ and D1 in W2 remained almost unchanged toward L-Leu, L-Val, L-Arg, and L-Glu (Figure 4B-4G), and so was their catalytic efficiency. 

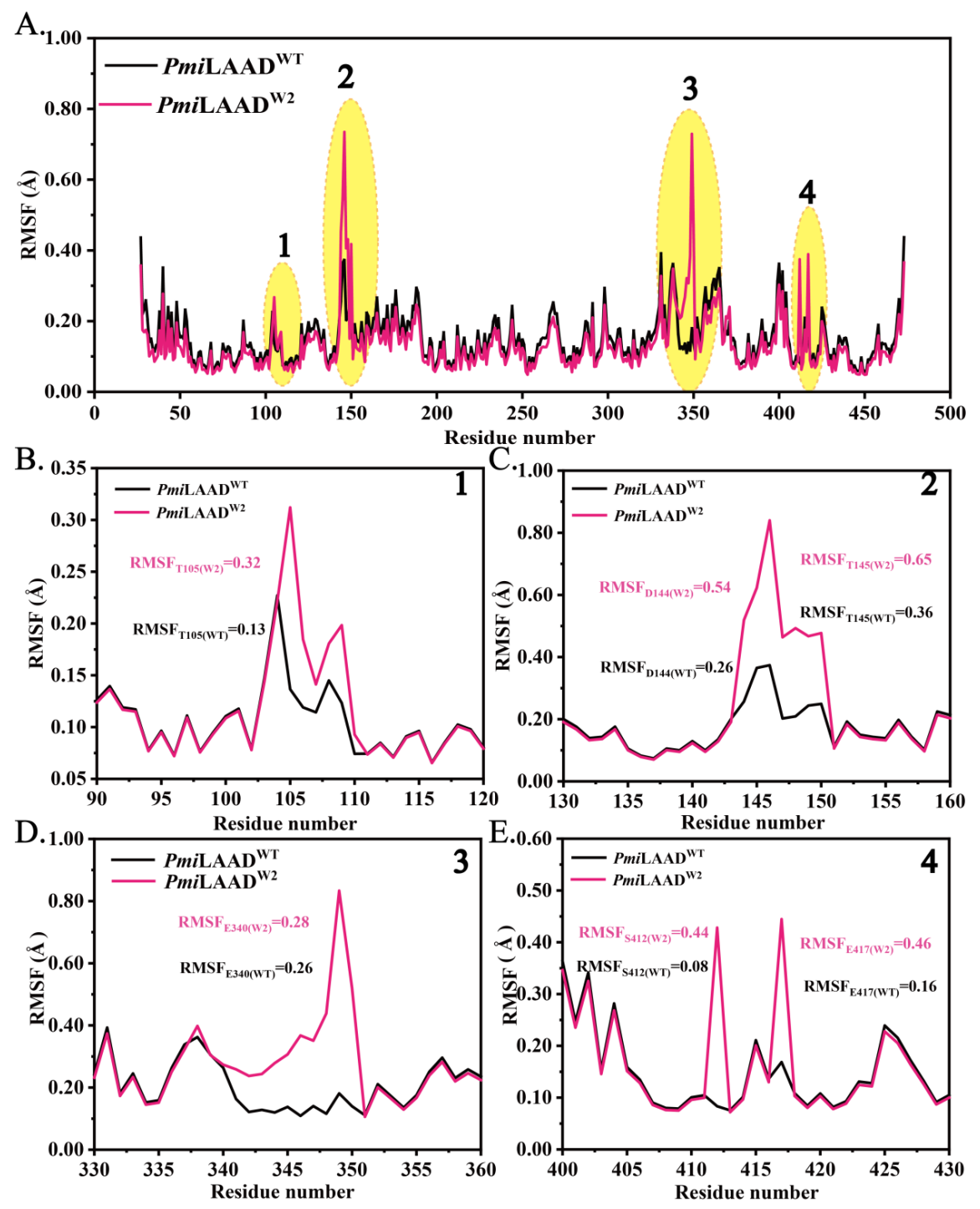

Figure 5. MD simulations of Pmi LAAD ${ }^{\mathrm{WT}}$ and variant W2. (A) Differences in the RMSFs of thePmi $\mathrm{LAAD}^{\mathrm{WT}}$ and variant $\mathrm{W} 2$; The yellow shadows represented the changes of the regions with noticeable movements. (B-E) Local analysis diagram of dynamic instability region.

\section{Construction of a high-performance universal catalyst}

To obtain a high-performance universal catalyst, W1 and W2 were combined into variant W3 (A variant that combines W1 and W2 mutation sites). However, contrary to expectations, the catalytic efficiency of W3 toward L-Leu, L-Met, L-Val, L-Phe, L-Arg, and L-Glu decreased by $79.7 \%, 60.2 \%, 50.4 \%, 40.3 \%, 15.6 \%$, and $5.8 \%$, respectively (Figure $4 \mathrm{~A}$ ). To address this drawback, a one-pot two-enzyme cascade strategy was applied, whereby W1 and W2 were co-expressed in E. coli (Figure 6). The newly constructed S1 strain benefited from the advantages provided by both W1 and W2, and exhibited better catalytic performance than Pmi LAAD. Specifically, conversion of L-Val, L-Arg, and L-Glu was increased by $20.8 \%, 131.3 \%$, and $487.4 \%$, respectively, compared to the wild-type enzyme; whereas conversion of L-Phe and L-Met was only $15.3 \%$ and $10.7 \%$ higher (Figure $6 \mathrm{H}$ ). The catalytic activity of $\mathrm{W} 2$, which has a preference for L-Phe and L-Met, constituted the limiting factor and required enhancement to improve overall efficiency of the whole- 
cell catalyst. To this end, the level of W2 was optimized by expressing two to four copies of W2 behind ribosome binding site (RBS) connectors. Among the newly generated strains S2, S3, and S4, respectively (Figure 6D-6G), S3 exhibited the best transformation performance, with $>90 \%$ conversion toward all of the six selected substrates.

The substrate scope of S3 was analyzed for 13 different amino acids $(100 \mathrm{~g} / \mathrm{L})$. Conversion of L-Met L-Val, L-Phe, L-Arg, L-Asp, and L-Glu was improved by $20.4 \%, 26.3 \%, 36.6 \%, 62.2 \%, 63.3 \%$, and $81.5 \%$ compared with the wild type; whereas conversion of L-Trp, L-Tyr, L-Ala, L-Ser, and L-Thr was increased by $23.0 \%$, $21.5 \%, 20.7 \%, 5.9 \%$, and $5.6 \%$, respectively (Figure 7). Conversion of L-Leu and L-Ile remained $>99 \%$.
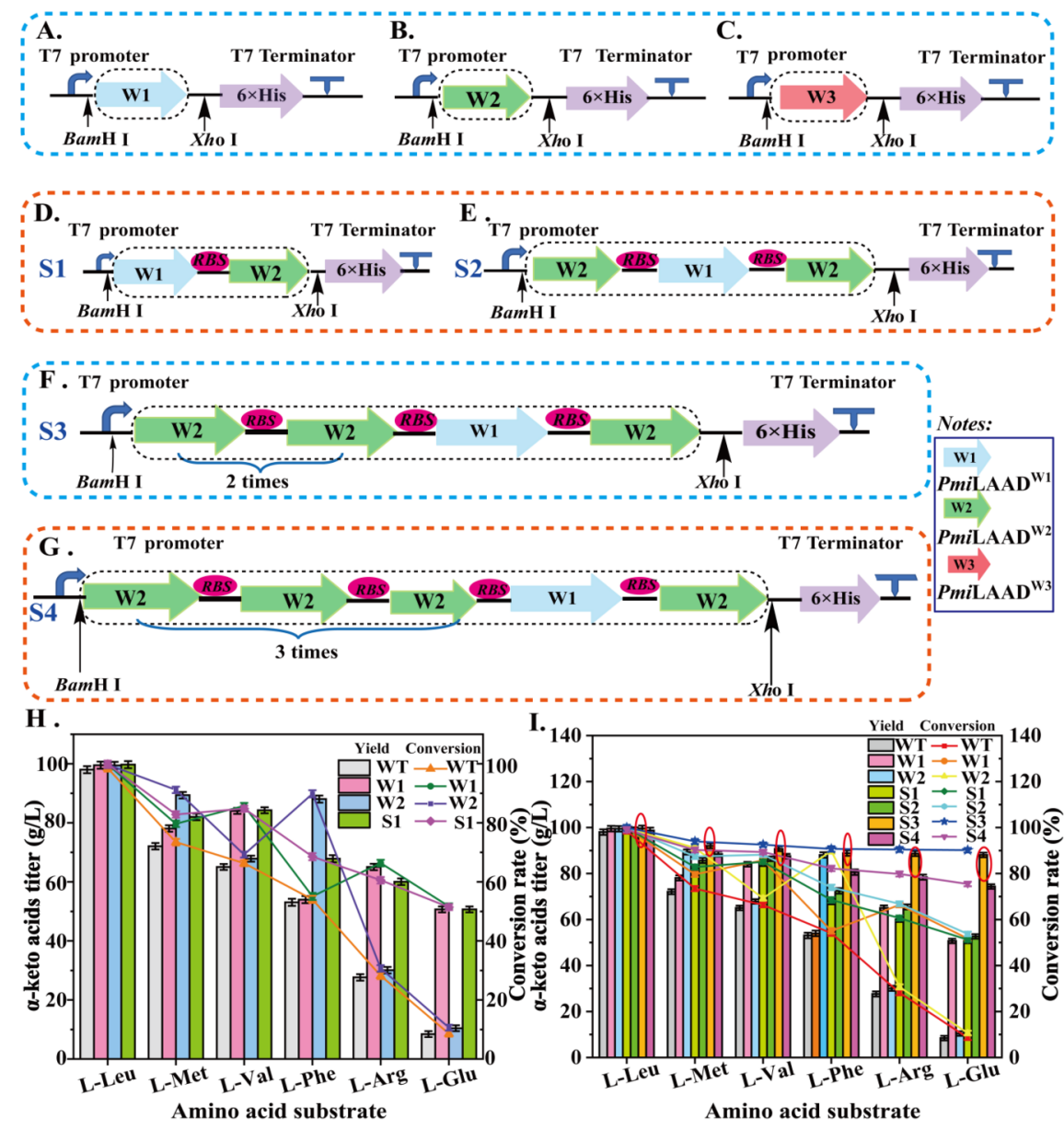

Figure 6. Schematic diagram of recombinant bacterial strain construction and transformation results. (AC) Schematic diagram of variant (W1, W2, and W3); (D-G) Schematic diagram of cascade system strains (S1, S2, S3, and S4); (H, I) Pmi LAAD ${ }^{\mathrm{WT}}$, variants (W1, W2 and W3) and strains (S1, S2, S3, and S4) conversion of representative amino acids to produce the corresponding $\alpha$-keto acids. 


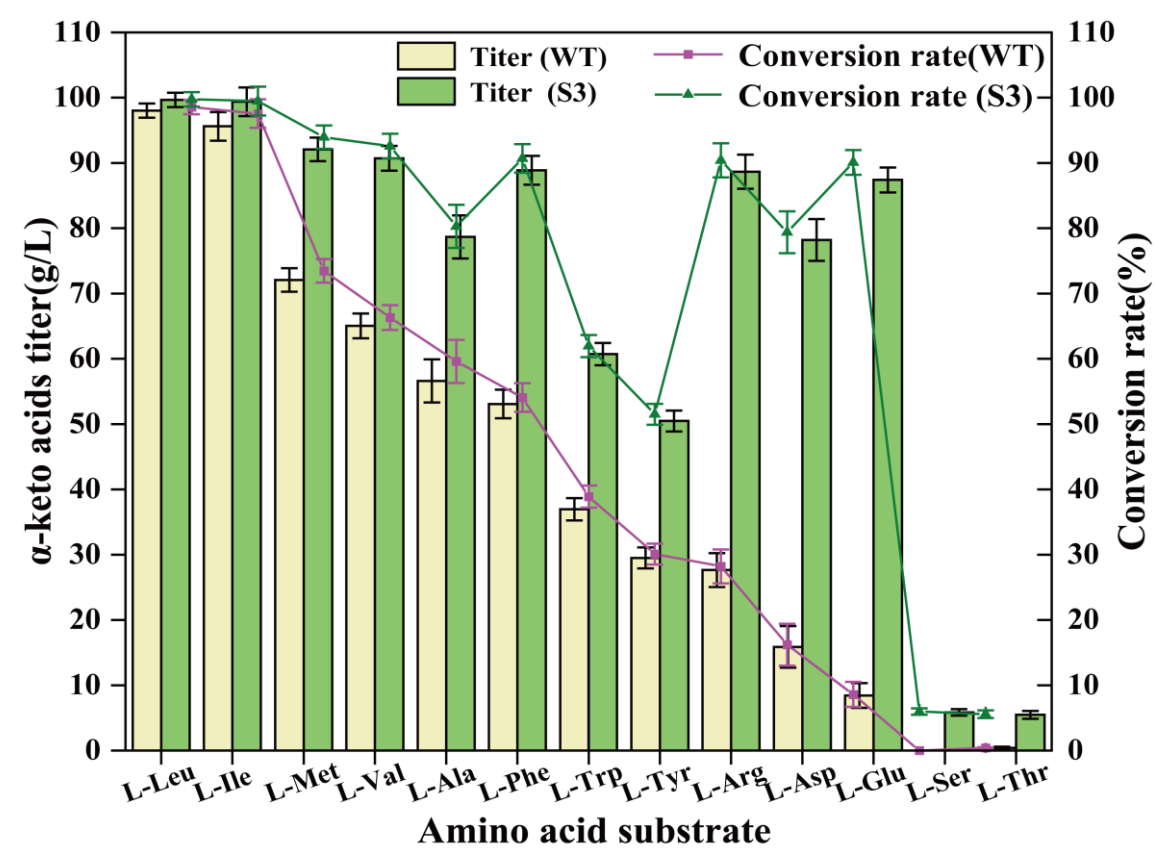

Figure 7. Analysis of 13 substrates conversion by strain S3.

A comparison of WT, W1, W2, and S3 (Table 1), revealed that all enzymes displayed high catalytic efficiency toward long-chain aliphatic amino acids (e.g., L-Leu and L-Ile). W1 exhibited good catalytic efficiency toward smaller amino acids (e.g., L-Val and L-Ala) and charged amino acids (e.g., L-Arg, L-Asp, and L-Glu); whereas W2 preferred large aromatic amino acids (e.g., L-Phe, L-Trp, and L-Tyr) and sulfur-containing L-Met. Most importantly, S3 showed elevated catalytic efficiency toward all the selected amino acids.

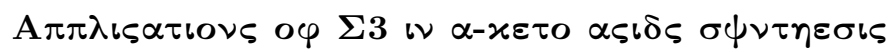

Considering the above results, preparative-scale transformation experiments were carried out using S3 as a whole-cell catalyst and L-Leu, L-Met, L-Val, and L-Phe as selected substrates. First, conditions were optimized in 250-mL shake flasks (Supplementary Figure 8 and 9), and 90-99\% conversions were obtained. The reactions were then scaled to fit a 5-L reactor, allowing for $101.3-109.8 \mathrm{~g} / \mathrm{L} \alpha$-keto acids to be produced from $110 \mathrm{~g} / \mathrm{L}$ of the corresponding substrates, achieving 93.7-99.9\% conversion rates and 101.4-202.6 g/L/d space-time yields (STY) in 12-24 h. The highest conversion was achieved using L-Leu (99.9\%); it amounted to $109.8 \mathrm{~g} / \mathrm{L}$ yield and $146.4 \mathrm{~g} / \mathrm{L} / \mathrm{d}$ STY. In contrast, the shortest conversion time $(12 \mathrm{~h})$ was achieved with L-Phe $(94.6 \%)$, which also resulted in the highest STY $(206.2 \mathrm{~g} / \mathrm{L} / \mathrm{d})$, a $285.4 \%$ increase compared to Pmi LAAD (Figure 8). 

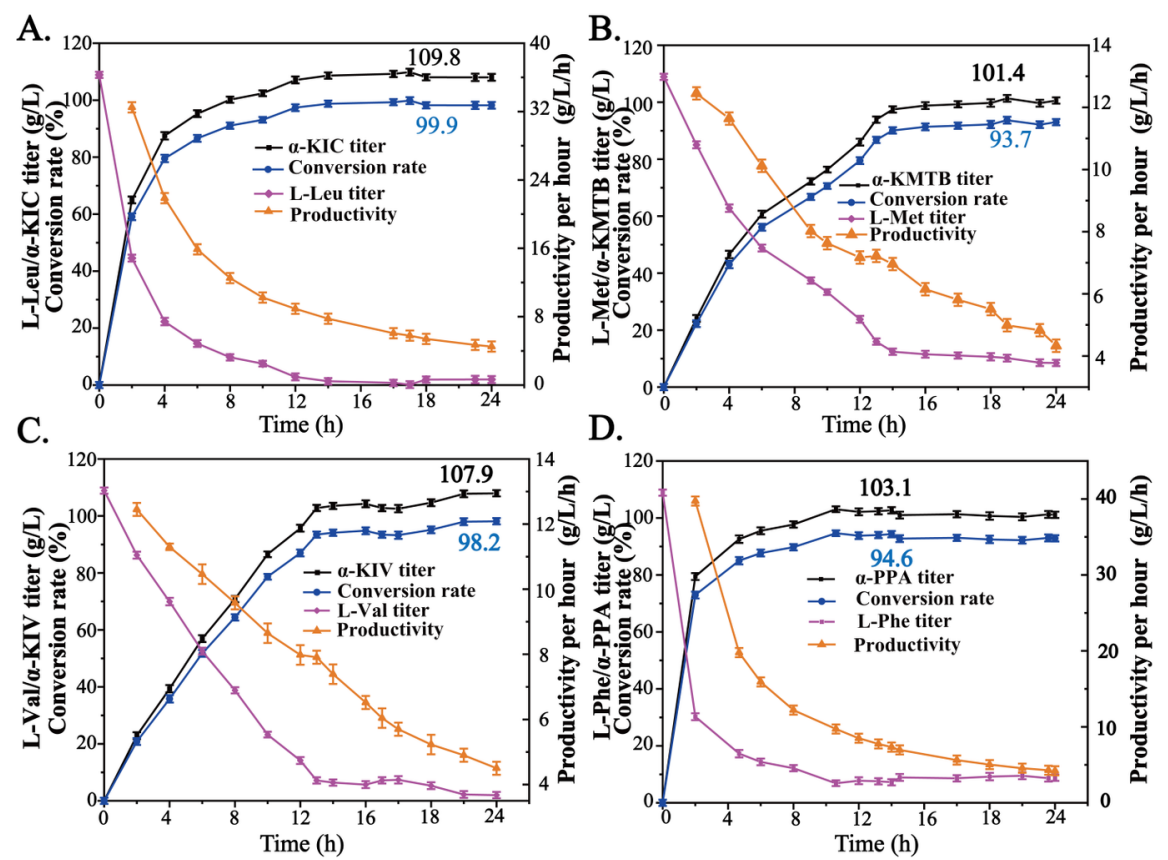

Figure 8. The conversion process curve of four key substrate L-amino acids using S3 in a 5-L fermentor.

\section{DISCUSSION}

In this study, an engineered $\alpha$-keto acid-producing Pmi LAAD recombinant strain with enhanced catalytic efficiency toward amino acid substrates harboring different side chains is reported. First, combined with the catalytic mechanism of Pmi LAAD, the $\vartheta$ and catalytic distance D1 were identified as the key factor affecting catalytic efficiency. Second, a mutational strategy to change the angle between $\alpha$-hydrogen and isoalloxazine ring and shorten D1 and increase catalytic efficiency was proposed, leading to two improved variants (W1 and W2). Third, strain S3 was generated by co-expressing W1 and W2 in E. coli . This last variant exhibited high catalytic efficiency toward all the selected amino acids. In summary, these results describe a protein engineering strategy for improving the catalytic efficiency of Pmi LAAD or other enzymes that share a similar mechanism and participate in $\alpha$-keto acid synthesis.

Understanding the catalytic mechanism of a reaction is of great significance while performing enzyme engineering. Existing reports on the catalytic mechanism of LAADs have described the overall reaction process, without explaining how protons are transferred between the substrate and FAD(Molla et al. 2017; Rosini et al. 2017). Here, we found that an hydride ion of substrate was required for the transfer of $\alpha \mathrm{C}-\mathrm{H}$ to FAD $\mathrm{N}(5)$, and the distance D1 was identified as the key factor affecting this step, as well as overall Pmi LAAD catalytic efficiency. Thus, our results revealed the catalytic mechanism of LAAD and provided effective guidance for the following protein engineering steps.

Shortening the catalytic distance D1 improved LAAD efficiency. Previously, the engineering of LAAD had focused on structural alignment(Wu et al. 2020), H-bond analysis(Pei et al.2020), pocket volume, and steric hindrance(Yuan et al. 2019), with the intent of minimizing product inhibition or maximizing catalytic efficiency. For example, Pei et al. reduced product inhibition caused by $\alpha$-keto valine $\left(K_{P I}\right.$-Val $)$ from 0.8 to $5.4 \mathrm{mM}$ by changing the H-bond interaction to generated variant $P m i$ LAAD ${ }^{\mathrm{S} 98 \mathrm{~A} / \mathrm{T} 105 \mathrm{~A} / \mathrm{S} 106 \mathrm{~A} / \mathrm{L} 341 \mathrm{~A}}$ (Pei

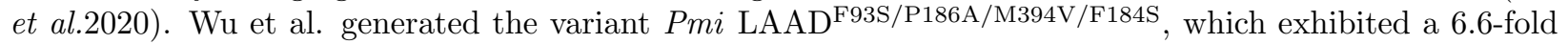
improvement in specific activity toward L-Phe(Wu et al. 2020). In contrast, in the present study, the protein engineering strategy was based on analysis of the underlying catalytic mechanism and, hence, on shortening of D1. Indeed, this strategy has proven to be very effective at improving LAAD catalytic efficiency. In 
future investigations, more biological tools, such as X-ray structures of the enzyme-substrate complexes, can be used to further understand the relationship between substrate specificities and the D1 distance and the mechanisms that enhance the LAAD efficiency.

The concept of multi-enzyme cascade was used to combine W1 and W2 variants in a single, highly efficient, and universal whole-cell catalyst. This whole-cell catalyst was constructed to be used in industrial applications to produce $\alpha$-keto acids. This multi-enzyme cascade was necessary when an enzyme was used to efficiently catalyze all substrates. Multi-enzyme cascades are often used in multi-step reactions(Qian et al. 2020; Shi et al. 2018). However, in the present study, W1 and W2, which catalyze the same deamination reaction, were cascaded simultaneously to improve the catalytic efficiency and substrate specificity of the same enzyme in a one-pot strategy. As proven here, this cascade method can be used to fine-tune the same reaction, whereby one variant is used to catalyze part of the reaction that the other variant cannot complete. Furthermore, this cascade method could replace conventional combination mutations, wherein the pairing of successful single mutated residues does not produce the expected result.

In summary, the biocatalytic process used in this study provides an attractive strategy to facilitate the industrial production of $\alpha$-keto acids. Furthermore, the proposed approach based on shortening of the catalytic distance and a multi-enzyme cascade reaction, offers an attractive strategy and reference for a more widespread application of this technique.

\section{ASSOCIATED CONTENT}

\section{Supporting Information}

Full experimental procedures, and Supplementary Tables and Figures (PDF).

Notes

The authors declare no competing financial interest.

\section{ACKNOWLEDGMENTS}

This work was financially supported by the National Natural Science Foundation of China (grants 22008089, 21878126), the Provincial Natural Science Foundation of Jiangsu Province (grant BK20200622), the key technologies R \& D Program of Jiangsu Province (grant BE2018623), and the National First-Class Discipline Program of Light Industry Technology and Engineering (grant LITE2018-20).

\section{ABBREVIATIONS}

$\mathrm{FAD}$, flavin adenosine dinucleotide; $\mathrm{FADH}^{-}$, the anionic form of reduced FAD; D1, Catalytic distance, i.e., the distance between substrate $\alpha \mathrm{C}-\mathrm{H}$ and FAD N(5); $K_{P I}$, Product inhibition constant; KIV, $\alpha$-keto valine; KMTB, $\alpha$-Keto- $\gamma$-methylthiobutyric acid; epPCR, error-prone polymerase chain reaction.

\section{REFERENCES}

Asano Y, Yasukawa K. 2019. Identification and development of amino acid oxidases. Current Opinion in Chemical Biology 49 : 76-83.

Ayodele BV, Alsaffar MA, Mustapa SI. 2020. An overview of integration opportunities for sustainable bioethanol production from first- and second-generation sugar-based feedstocks. Journal Cleaner Production $245: 20-68$.

Cooper AJ, Ginos JZ, Meister A. 1983. Synthesis and properties of the $\alpha$-keto acids. Chemical Reviews 83 : 321-358.

Hossain GS, Li J, Chen J. 2014a. L-amino acid oxidases from microbial sources: types, properties, functions, and applications. Applied Microbiology Biotechnology 98 (4): 1507-1515. 
Hossain GS, Li J, Chen J. 2014b. One-step biosynthesis of $\alpha$-Keto- $\gamma$-methylthiobutyric acid from Lmethionine by an Escherichia coli whole-cell biocatalyst expressing an engineered L-amino acid deaminase from Proteus vulgaris. Plos One 9 (12): 16.

Jambunathan P, Zhang KC. 2014. Novel pathways and products from 2-keto acids. Current Opinion Biotechnology $29: 1-7$.

Jin L, Zhang X-L, Wang Y-Q. 2021. Palladium-catalyzed dehydrogenative fluoroalkoxylation of benzaldehydes. Organic letters 23 (5): 1921-1927.

Ju Y, Liu Z, Zhou H. 2017. Membrane binding of the insertion sequence ofProteus vulgaris L-amino acid deaminase stabilizes protein structure and increases catalytic activity. Scientific Reports 7 : 1-11.

Ju Y, Tong S, Zhou H. 2016. Crystal structure of a membrane-bound L-amino acid deaminase from Proteus vulgaris. Journal Structural Biology 195 (3): 306-315.

Li PG, Zhu H, Zou LH. 2019. Copper-catalyzed coupling of anthranils and $\alpha$-keto acids: direct synthesis of a-ketoamides. Organic \& Biomolecular Chemistry 17 (24): 5902-5907.

Li R, Hossain G, Liu L. 2017. Rational molecular engineering of L-amino acid deaminase for production of $\alpha$-ketoisovaleric acid from L-valine by Escherichia coli . Rsc Advances 7 (11): 6615-6621.

Liu L, Hossain G, Chen J. 2013. One-step production of $\alpha$-ketoglutaric acid from glutamic acid with an engineered L-amino acid deaminase fromProteus mirabilis . Journal of Biotechnology 164 (1): 97-104.

Liu K, Gong M, Liu L. 2020. Biotransformation and chiral resolution of D,L-alanine into pyruvate and D-alanine with a whole-cell biocatalyst expressing L-amino acid deaminase. Biotechnology and Applied Biochemistry 9 (1): 668-676.

Manjarin R, Boutry-Regard C, Davis TA. 2020. Intermittent leucine pulses during continuous feeding alters novel components involved in skeletal muscle growth of neonatal pigs. Amino Acids 52 (9): 1319-1335.

Mattevi A, Vanoni MA, Todone F, Rizzi M, Teplyakov A, Coda A, et al. 1996. Crystal structure of D-amino acid oxidase: a case of active site mirror-image convergent evolution with flavocytochrome b(2). Proceedings of the National Academy of Sciences of the United States of America 93 (15): 7496-7501.

Molla G, Melis R, Pollegioni L. 2017. Breaking the mirror: L-amino acid deaminase, a novel stereoselective biocatalyst. Biotechnology Advances 35 (6): 657-668.

Motta P, Molla G, Nardini M. 2016. Structure-function relationships in L-amino acid deaminase, a flavoprotein belonging to a novel class of biotechnologically relevant enzymes. Journal Biological Chemistry 291 (20): 10457-10475.

Nakano S, Minamino Y, Hasebe F, Ito S. 2019. Deracemization and stereoinversion to aromatic D-amino acid derivatives with ancestral L-amino acid oxidase. ACS Catalysis 9 (11): 10152-10158.

Ogo S, Uehara K, Abura T, Fukuzumi S. 2004. pH-dependent chemoselective synthesis of $\alpha$-amino acids. Reductive amination of $\alpha$-keto acids with ammonia catalyzed by acid-stable iridium hydride complexes in water.Journal American Chemical Society 126 (10): 3020-3021.

Pei S, Ruan X, Liu J, Song W, Chen X, Luo Q, et al. 2020. Enhancement of $\alpha$-ketoisovalerate production by relieving the product inhibition of L-amino acid deaminase from Proteus mirabilis .Chinese Journal of Chemical Engineering 28 (8): 2190-2199.

Qian Y, Lu C, Liu J, Song W, Chen X, Luo Q, et al. 2020. Engineering protonation conformation of Laspartate- $\alpha$-decarboxylase to relieve mechanism-based inactivation. Biotechnology Bioengineering 117 (6): $1607-1614$.

Rosini E, Melis R, Pollegioni L. 2017. Deracemization and stereoinversion of $\alpha$-amino acids by L-amino acid aeaminase.Advanced Synthesis Catalysis 359 (21): 3773-3781. 
Sandoval BA, Clayman PD, Oblinsky DG, Oh S, Nakano Y, Bird M, et al. 2021. Photoenzymatic reductions enabled by direct excitation of flavin-dependent "Ene"-reductases. Journal American Chemical Society 143 (4): 1735-1739.

Shi JF, Wu YZ, Zhang SH, Tian Y, Yang D, Jiang ZY. 2018. Bioinspired construction of multi-enzyme catalytic systems. Chemical Society Reviews, 47 (12): 4295-4313.

Song W, Xu X, Gao C, Zhang YX, Wu J, Liu J, et al. 2020. Open gate of Corynebacterium glutamicum threonine deaminase for efficient synthesis of bulky $\alpha$-keto acids. ACS Catalysis 10 (17): 9994-10004.

Song Y, Li JH, Shin HD, Liu L, Du GC, Chen J. 2016. Biotechnological production of $\alpha$-keto acids: Current status and perspectives.Bioresource Technology 219 : 716-724.

Taylor PP, Pantaleone DP, Senkpeil RF, Fotheringham IG. 1998. Novel biosynthetic approaches to the production of unnatural amino acids using transaminases. Trends Biotechnology 16 (10): 412-418.

Umhau S, Pollegioni L, Molla G, Diederichs K, Welte W, Pilone MS, et al. 2000. The x-ray structure of D-amino acid oxidase at very high resolution identifies the chemical mechanism of flavin-dependent substrate dehydrogenation. Proceedings of the National Academy of Sciences of the United States of America 97 (23): 12463-12468.

Vogt M, Haas S, Polen T, van Ooyen J, Bott M. 2015. Production of 2-ketoisocaproate with Corynebacterium glutamicum strains devoid of plasmids and heterologous genes. Microbial Biotechnology 8 (2): 351-360.

Wakamatsu T, Sakuraba H, Ohshima T. 2017. Structural insights into L-tryptophan dehydrogenase from a photoautotrophic Cyanobacterium Nostoc punctiforme. Applied Environmental Microbiology 83 (2): 1-48.

Wang M, Xu HZ, Yao Y. 2019. Compound $\alpha$-keto acid tablet supplementation alleviates chronic kidney disease progression via inhibition of the NF-kB and MAPK pathways. Journal of Translational Medicine 17 (122): 2-15.

Williams A, Rayson M, Montgomery H. 2000. Physiology - The ACE gene and muscle performance. Nature 403 (6770): 614-614.

Wu, Guo X, Liu Z. 2020. Efficient enzymatic synthesis of $\alpha$-keto acids by redesigned substrate-binding pocket of the L-amino acid deaminase (Pmi LAAD). Enzyme and microbial technology 132 : 109393-109399.

Wu Y, Zha MS, Sun BG. 2018. Novel method for L-methionine production catalyzed by the aminotransferase ARO8 from Saccharomyces cerevisiae. Journal Agricultural Food Chemistry 66 (24): 6116-6122.

Xie C, Song JL, Wu HR, Hu Y, Liu HZ, Zhang ZR, et al. 2019. Ambient reductive amination of levulinic acid to pyrrolidones over $\mathrm{Pt}$ nanocatalysts on porous $\mathrm{TiO}_{2}$ nanosheets. Journal of the American Chemical Society 141 (9): 4002-4009.

Yang B, Wang H, Song W, Chen X, Liu J, Luo Q, et al. 2017. Engineering of the conformational dynamics of lipase to increase enantioselectivity. ACS Catalysis 7 (11): 7593-7599.

Yuan Y, Song W, Liu J, Chen X, Luo Q, Liu L. 2019. Production of $\alpha$-etoisocaproate and $\alpha$-keto- $\beta$ methylvalerate by engineered L-amino acid deaminase. ChemCatChem 11 (10): 2464-2472.

Zhao HY, Yu RH, Qiao H, Liu CL. 2020. Study on the formation of glycine by hydantoin and its kinetics. ACS Omega 5 (23): 13463-13472. 